Communications in Physics, Vol.21, No. 4 (2011), pp. 373-377

\title{
INVESTIGATION OF ENERGY TRANSFER IN A BLEND OF ELECTROLUMINESCENT CONDUCTING POLYMERS
}

\author{
NGUYEN NANG DINH, NGUYEN PHUONG HOAI NAM, AND DO NGOC CHUNG \\ Faculty of Engineering Physics and Nanotechnology, \\ University of Engineering and Technology, Vietnam National University, Hanoi
}

\begin{abstract}
With the aim of improving the photonic efficiency of an organic light emitting diode a blend of electroluminescent polymers, poly[9-vinylcarbarzole] (PVK) and poly[2-methoxy-5-(2ethylhexyloxy)-1,4-phenylenevinylene] (MEH-PPV) was prepared. The energy transfer in the blend was investigated through the comparison of absorption, photoluminescence (PL) and currentvoltage $(I-V)$ characteristics of the blend and standard polymers. The obtained results showed that energy transfer from PVK to MEH-PPV enable to form localized excited-state complexes (e.g. exciplexes) in MEH-PPV. The PL intensity of the blended polymers was enhanced as the relative content of $M E H-P P V$ was increased, and particularly, the highest improved $P L$ was observed for an weight fraction of $15 \mathrm{wt} \%$ of MEH-PPV in PVK. Organic light emitting diodes made from these blends would exhibit a large photonic efficiency.
\end{abstract}

\section{INTRODUCTION}

Since the discovery of electroluminescence (EL) of organic molecules [1], rapid progress has been made in research on these materials due to their potential applications in flat panel display. The EL devices based on organic small molecules or polymers have advantages compared with inorganic EL devices in such properties as lower operating voltage and faster response time. Among a number of organic EL devices, polymers molecularly doped with highly efficient fluorescent dopants are important in obtaining high luminescence efficiency. They effectively prevent the crystallization or aggregation of the small molecules [2]. The blend compositions containing mixtures of electron transporting and emitting materials are often used to fabricate efficient single-layer devices with high quantum efficiencies [3,4]. Kido et al. [5] demonstrated that molecularly doped polymers provide a high flexibility in designing EL devices, in which poly(9-vinylcarbazole) (PVK) was used both as a matrix and as a hole transport material. Poly[2-methoxy-5(2-ethylhexyloxy)-1,4-phenylenevinylene] (MEH-PPV) has been a subject of intense investigation because it can be dissolved in many organic solvents which allows MEH-PPV to be easily processed into various types $[6,7]$. It is well-known that this polymer shows yellowish-red light when it is processed into electroluminescence devices, and the different quantum efficiencies were obtained by changing the work function of the electrode $[8,9]$.

In this paper, the blend films of PVK and MEH-PPV with different weight fraction of MEH-PPV/PVK have been fabricated and characterized. The photoluminescence (PL) measurement indicated that there is an efficient energy transfer between PVK and MEH-PPV. The energy-transfer process from PVK to MEH-PPV was observed, and thus 
the emission of MEH-PPV was exclusively observed when the blended polymer film was photoexcited by light whose energy was corresponding to the absorption of PVK. Moreover, the relative PL quantum efficiency increased as the weight fraction in the blended polymer increased. The current-voltage (I-V) characteristics were also studied.

\section{EXPERIMENTAL}

Figure 1 shows the molecular structures of PVK and MEH-PPV used in this study. PVK and MEH-PPV were purchased from Aldrich Chemical Ltd. and used as received. Indium tin oxide (ITO) and $\mathrm{Al}$ were used as the anode and the cathode, respectively. The sheet resistance of the ITO was $15 \Omega /$ ? . Before using, the ITO substrate and glass were cleaned carefully according to the method of Tang and Van Slyke [1]. The blends were obtained by mixing MEH-PPV and PVK by the weight fraction of the two polymers as $0.05,0.10,0.15$, and 0.20 , in 1,2 dichloroethane (ca. $13 \mathrm{mg} / \mathrm{ml}$ ). The blends were spincoated onto the substrates and dried in vacuum at $80{ }^{\circ} \mathrm{C}$ for two hours. The thickness of the polymer layer was controlled both by spin speed and by the concentration of polymer in the solvent. The surface morphology of samples was investigated by using a "Hitachi" Field Emission Scanning Electron Microscopy (FE-SEM) S-4800. The thicknesses of the films were measured to be around $120 \mathrm{~nm}$. FE-SEM images showed no indication of the phase separation or the layer formation due to the immiscibility of two polymers (Fig. 2). The absorption spectra of the coated films were recorded by a Jasco UV-VIS-NIR V570 spectrometer. The PL spectra were carried-out by using a FL3-2 spectrophotometer and I-V characteristics were measured on an Auto-Lab Potentiostat PGS-30. All the photophysical measurements were performed in air, at room temperature.
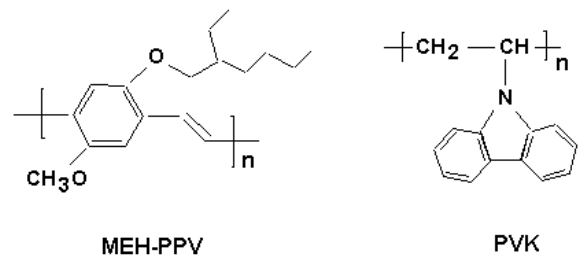

Fig. 1. Molecular structures of the electroluminescent polymers (MEH-PPV/ PVK) which were used in this work.

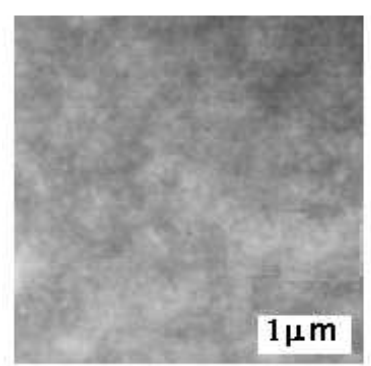

(a)

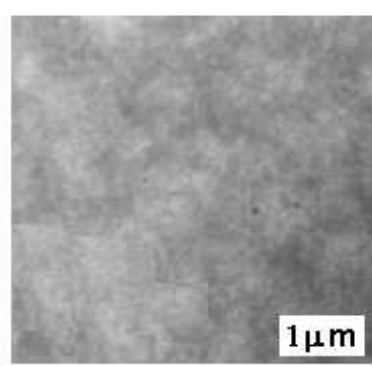

(b)

Fig. 2. FE-SEM of a pure PVK (a) and a blend with the weight fraction of MEH-PPV /PVK of 0.15.

\section{RESULTS AND DISCUSSION}

Figure 3 compares the UV-Vis and PL spectra of bulk films of PVK and MEHPPV. The PL emission from PVK film excited at $325 \mathrm{~nm}$ overlaps with the absorption peaks of MEH-PPV, and, thus an efficient Förster energy transfer can be anticipated [10]. According to Fig. 2, PVK absorbs from 200 to $350 \mathrm{~nm}$ with two picks at 295 and $339 \mathrm{~nm}$, 
respectively and emits at $410 \mathrm{~nm}$. In contrast, MEH-PPV reveals a broad absorption in the longer wavelength region of 420 to 520 and maximum absorption at $490 \mathrm{~nm}$ arising from the $\pi$-conjugated structure [11]. MEH-PPV is the yellowish-red light emitters and the PL spectra of the thin film show an emission peak at $593 \mathrm{~nm}$ and the other at 640 and small shoulder at $707 \mathrm{~nm}$ reflecting excimer formation. From Fig. 4 the blended polymer films showed the absorption peaks corresponding to both PVK and MEH-PPV, and their intensities were varied depending on the weight fractions of the two polymers.

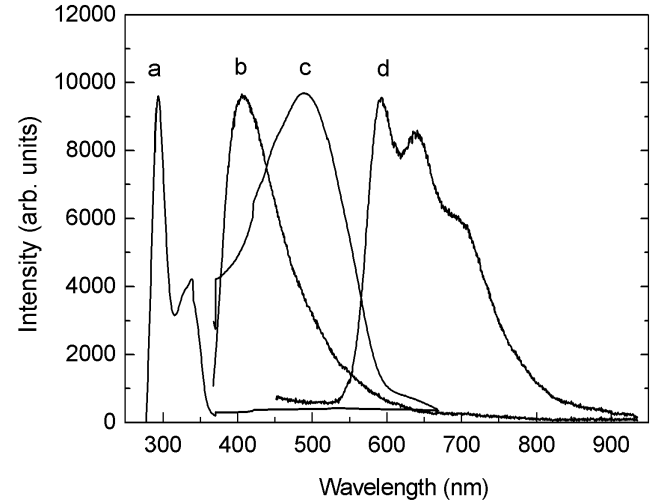

Fig. 3. Molecular structures of the electroluminescent polymers (MEH-PPV/ PVK) which were used in this work.

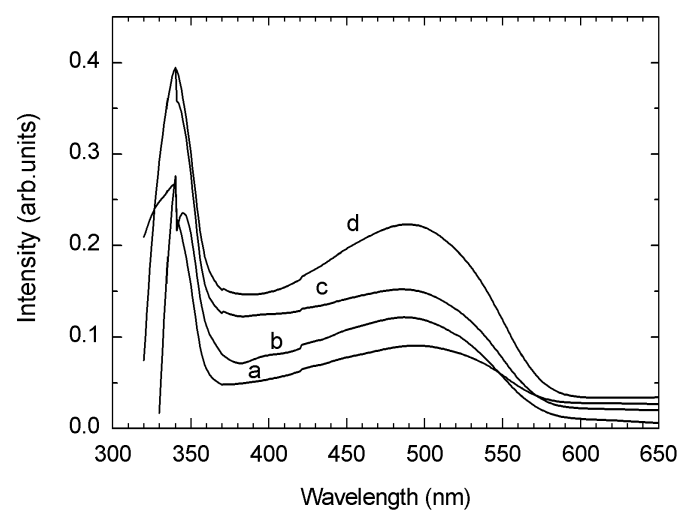

Fig. 4. Optical absorption spectra of (a) 0.05, (b) 0.10 , (c) 0.15 , (d) 0.20 blended polymers of MEH-PPV and PVK.

Figure 5 shows the PL spectra of PVK and the blend films which were excited at $325 \mathrm{~nm}$. From this figure, one can see that the peaks at 410 and $555 \mathrm{~nm}$ belong to PVK and MEH-PPV emissions, respectively. Though PVK concentration is much higher than that of MEH-PPV, the PL spectra are dominated by the green-yellow MEH-PPV emission. With the increase of MEH-PPV concentration in the blends, the PVK fluorescence decreases indicating that more energy transfer occurs from PVK to MEH-PPV. At the weight fraction of MEH-PPV/ PVK=0.15 and 0.20, the efficient Förster energy transfer from PVK to MEH-PPV becomes saturated, which results in the appearance of MEHPPV emission in the green region. Further increase in the degree of doping diminishes the PL intensities from the MEH-PPV. Such results are common in blend systems where energy transfer takes place [12-14] and can be explained by the following process. At lower concentrations of MEH-PPV, the energy transfer from PVK to MEH-PPV is incomplete because the average distance from a photoexcited polymer chain to the nearest $\mathrm{MEH}-\mathrm{PPV}$ molecule is too large. At higher concentrations, more energies are transferred from the matrix to MEH-PPV molecules, but the fluorescence efficiency is reduced by concentration-quenching [15]. Fig. 6 shows the PL spectra of the blended polymer films with an excitation of $442 \mathrm{~nm}$ to examine the blending weight fraction dependence on the MEHPPV emission intensity. MEH-PPV strongly absorbs the light at $442 \mathrm{~nm}$, but PVK does not (Fig. 3). It has been also reported that the emission intensity is enhanced as the emitting chromophore is diluted by other inert polymers in solid state [16]. The 
emission intensity was increased as MEH-PPV was diluted by PVK, which is consistent with the previous results [16]. It can be explained by the fact that the nonradiative decays, especially intermolecular quenching, were reduced by the dilution. Therefore, one can conclude that the radiative decay of MEH-PPV through energy transfer and internal excitation increased as MEH-PPV was diluted.

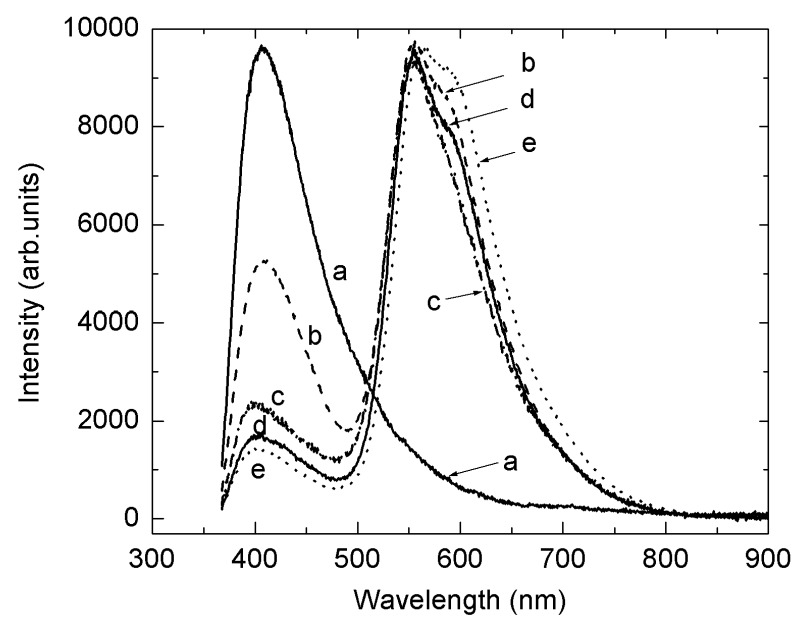

Fig. 5. Normalized photoluminescence spectra of (a) apure PVK film and of the blends with different weight fraction: 0.05 (b), 0.10 (c), 0.15 (d) 0.15 and 0.20 . All PL spectra were recorded with an excitation at $325 \mathrm{~nm}$.

The single EL-devices based on the blend polymer films were fabricated which consists of a transparent indium-tin-oxide (ITO) conducting electrode, a MEH-PPV/PVK blended film, and an aluminum (Al) electrode. The thickness of the blend film was estimated to be around $120 \mathrm{~nm}$. For the samples, D1, D2, D3 and D4 are abbreviated to the devices with the blends of the weight fraction corresponding to of $0.05,0.10,0.15$ and 0.20 , respectively. Fig. 7 shows the I-V characteristics of these devices. From Fig. 6, one can note that the device D3 reveals the best performance of an OLED, the turn-on votage is around $4 \mathrm{~V}$. This is because the inverse current of the device is almost eliminated and the from the turn-on voltage the voltage dependence of the current is almost vertical. This showed that the optimal weight fraction of the blend was ca. $15 \mathrm{wt} \%$.

\section{CONCLUSIONS}

A new emitting layer based on the blend of PVK and MEH-PPV was fabricated. The investigation of the photophysics of the blended polymers suggest that the excitons in PVK produced upon photoexcitation rapidly transfer to MEH-PPV. The energy-transfer process enhances not only the PL quantum efficiency of the blended polymers but also the EL quantum efficiency. The blend film with optimal ratio will be a candidate for a green light emitting diode. 


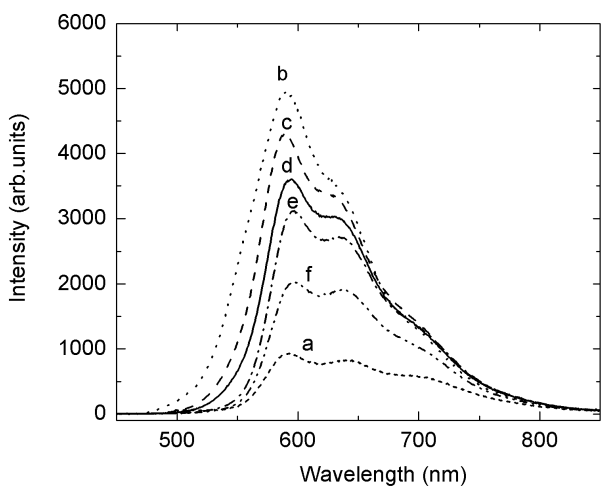

Fig. 6. Photoluminescence spectra of a pure MEH-PPV film (a) and of the blended films with the weight fraction of 0.05 (b) 0.10 (c), 0.15 (d), 0.20 (e) and 0.50 (f). All PL spectra of the films were recorded with an excitation at $442 \mathrm{~nm}$.

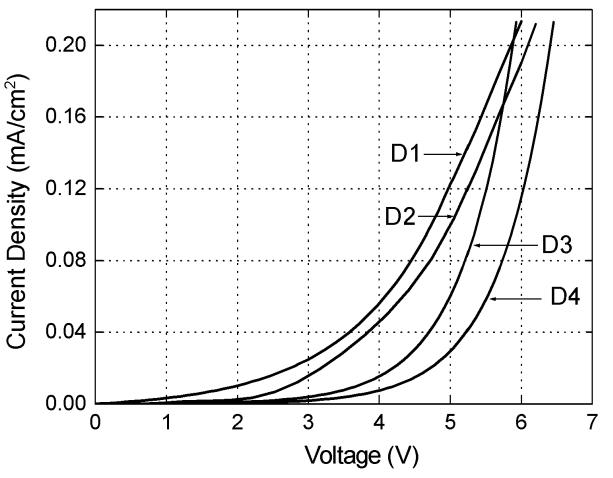

Fig. 7. Electrical properties of OLEDs: D1: ITO/PVK:MEH-PPV/Al (0.05), D2: ITO/PVK:MEH-PPV/Al (0.10), D3: ITO/PVK:MEH-PPV/Al (0.15), D4: ITO/PVK:MEH-PPV/Al (0.20)

\section{ACKNOWLEDGMENT}

This work was supported in part by the Vietnam National University, Hanoi in 2010 (Project Code: CN.10.08) and MOST of Vietnam (Project on Fundamental Scientific Research and Applications, Code: 1/2010/HD-DTNCCBUD).

\section{REFERENCES}

[1] C. W. Tang, S. A. Van Slyke, Appl. Phys. 51 (1987) 913.

[2] J. Kido, M. Kohda, K. Okuyama, K. Nagai, Appl. Phys. Lett. 61 (1992) 791.

[3] J.- I. Lee, I.- N. Kang, D.-H. Hwang, H.-K. Shim, S. C. Jeoung, and D. Kim, Chem. Mater. 8 (1996) 1925.

[4] S. H. Jin, W. H. Kim, I.S. Song, S. K. Kwon, K. S. Lee, and E. M. Han, Thin Solid Films 363 (2000) 255.

[5] J. Kido, K. Hongawa, K. Okuyama, K. Nagai, Appl. Phys. Lett. 64 (1994) 815.

[6] A. R. Brown, D. D. C. Bradley, J. H. Burroughes, R. H. Friend, N. C. Greenham, P. L. Burn, A. B. Holmes, and A. Kraft, Appl. Phys. Lett. 61 (1992) 2793.

[7] L. Smilowitz, A. Hays, A. J. Heeger, G. Wang, and J. E. Bowers, J. Chem. Phys. 98 (1993) 6504.

[8] Y. Yang and A. J Heeger, Appl. Phys. Lett. 64 (1994) 1245.

[9] I. D. Parker, J. Appl. Phys. 75 (1994) 1656.

[10] N. P. H. Nam, S. W. Cha, B.S. Kim, S.-H. Choi, D. S. Choi, J.-I. Jin, Synth. Met. 130 (2002) 271.

[11] S. J. Chung, J.-I. Jin, K.K. Kim, Adv. Mater. 9 (1997) 551.

[12] Y. Qiu, L. Duan, X. Hu, D. Zhang, M. Zheng, F. Bai, Synth. Met. 123 (2001) 41.

[13] T. Virgili, D.G. Lidzey, D.D.C. Bradley, Adv. Mater. 12 (2000) 58.

[14] T. W. Lee, O. Park, H.N. Cho, Y.Ch. Kim, Curr. Appl. Phys. 1 (2001) 364.

[15] M. D. McGehee, Tr. Bergstedt, Ch. Zhang, A.P. Saab, M.B. Oregan, G.C. Bazan, V.I. Srdanov, A.J. Heeger, Adv. Mater. 11 (1999) 1351.

[16] U. Lemmer, S. Karg, M. Scheidler, M. Deussen, W. Riess, B. Cleve, P. Thomas, H. Bässler, M. Schwoerer, and E. O. Göbel, Synth. Met. 67 (1994) 169. 